\title{
Repeat CRS/HIPEC: It Comes Down to Tumor Biology and Ability to Achieve a Complete CRS
}

\author{
Konstantinos I. Votanopoulos, MD, PhD \\ Department of Surgery, Division of Surgical Oncology, Medical Center Boulevard, Wake Forest University, Winston \\ Salem, NC
}

The concept of repeat cytoreductive surgery/hyperthermic intraperitoneal chemotherapy (CRS/HIPEC) in appendiceal primaries was developed as a necessity directly related to the natural history of debilitating peritoneal recurrences after initial cytoreduction. This is accurately depicted in the 1957-1983 historic Mayo experience, where appendiceal and ovarian patients were treated with repeat debulking operations followed by intraperitoneal ${ }^{32} \mathrm{P}$ or ${ }^{198} \mathrm{Au}$ radiotherapy, or intraperitoneal 5-fluorouracil and cyclophosphamide, in an effort to address residual peritoneal disease. Despite the fact that, at that time, physical examination was the most common method detecting disease recurrence, 5- and 10-year survival was reported as $53 \%$ and $32 \%$, respectively. ${ }^{1}$

The introduction of CRS/HIPEC along with modern imaging techniques dramatically improved survival outcomes, with expected 5-year survival routinely approaching $80 \%$ in low-grade appendiceal (LGA) primaries treated with $\mathrm{CC} 0-\mathrm{R} 0 / \mathrm{R} 1$ complete macroscopic cytoreduction. $^{2-5}$ Despite the efficacy of CRS/HIPEC, up to $70 \%$ of patients, especially those presenting with voluminous disease (Peritoneal Cancer Index [PCI] >20), will eventually recur. Although a smaller subset of patients will exhibit recurrences with slow or no progression, the majority, if left untreated, will succumb to bowel obstruction. Given the lack of response to chemotherapy by

This is a U.S. government work and not under copyright protection in the U.S.; foreign copyright protection may apply 2022

First Received: 3 January 2022

Accepted: 26 January 2022;

Published Online: 18 February 2022

K. I. Votanopoulos, MD, PhD

e-mail: kvotanop@wakehealth.edu the LGA primaries and the predominantly limited to the peritoneal cavity pattern of recurrence, up to $20 \%$ of patients will be offered repeat CRS/HIPEC.

One of the earliest series of repeat CRS/PIC (perioperative intraperitoneal chemotherapy) was published by Esquivel and Sugarbaker in 2001, introducing the concept of staged CRS/PIC procedures in patients with high-volume disease. A number of these repeat CRS/PIC cases were performed as scheduled second-look operations in patients presenting with neglected high-volume pseudomyxoma peritonei (PMP). These patients quite often present with depleted physiologic reserves and cannot sustain a single-stage surgical resection without a prohibitive risk of mortality. Of the initial $321 \mathrm{CRS} / \mathrm{HIPEC}$ patients, $79(24.6 \%)$ underwent a second-look operation inclusive of CRS/PIC. Complete cytoreduction at the second index case achieved an 84\% 5-year survival, compared with $68 \%$ for those patients who did not have repeat procedures. $^{6}$

A few years later, the same group analyzed patients who developed a recurrence after prior $\mathrm{CC}-0 / 1$ resection. They clearly demonstrated that the pattern of recurrence was also important, with diffuse peritoneal progression behaving much worse than isolated recurrence $(p=0.006)$, while complete CRS at the second operation was the only significant factor for extended survival. No patient was alive post 76 months without reoperation, demonstrating that the indolent behavior of these tumors will allow for long-term survival even after incomplete CRS, but not to the extent achieved by complete macroscopic CRS offered by a redo procedure. $^{7}$

In this issue of Annals of Surgical Oncology, LopezRamirez et al. elegantly demonstrate the natural course of the disease in cases where, for a number of reasons, a redo CRS/HIPEC was not attempted (control). ${ }^{8}$ Fifty-five repeat 
HIPEC procedures (36 LGAs, 13 high-grade, and 6 signet ring) treated over a span of 22 years were compared with 55 propensity matched patients based on age at recurrence, PCI, completeness of CRS, lymph node status, grade of primary, and time to recurrence as an indirect indication of tumor biology. Morbidity and mortality were comparable with initial CRS/HIPEC. Repeat CRS/HIPEC was resetting the clock for the LGA group, with an achieved 5-year survival of $81.3 \%$ versus $46.3 \%(p<0.001)$ for the control. In the high-grade primaries, the difference in the achieved 5-year survival was also in favor of repeat HIPEC ( 50 vs. $12 \% ; p=0.02$ ), while there were no 5 -year survivors for the signet ring cohort with or without reoperation.

The study is well aligned with prior work on the field and has similar limitations, including the underrepresentation of high-grade primaries as well as the inability of the current $\mathrm{CC}$ and $\mathrm{R}$ resection classification systems to define the exact volume of residual disease in cases of incomplete cytoreductions. ${ }^{9,10}$

It is not a coincidence that all published repeat CRS/ HIPEC series from centers with more than 20 years of experience include a modest number of high-grade appendiceal (HGA) primaries and an even smaller number of signet ring cells, clearly depicting the vast differences in biologic behavior between HGA and LGA cancer. Achieving a $\mathrm{CC} 0$ resection during a repeat $\mathrm{CRS}$ for an HGA primary is an even rarer event than attempting it, due to the infiltrative and often sclerotic nature of these tumors, protracted courses of systemic chemotherapy, prior surgical resections, and, often, marginal functional status. Intense fibrosis is also present with redo LGA primaries or neglected first-time CRSs that have been left undiagnosed evolving over many years, possibly suggesting a proinflammatory or an overactivated cancer-associated fibroblast (CAF) pathway within the peritoneal/stroma microenvironment. Particularly in the LGA subgroup, patients succumb to mechanical obstruction with excessive fibrotic tissue and a relatively scarce number of cancer epithelial cells. Delineating the interaction framework between the epithelial and CAF component or the stroma for appendiceal primaries will likely facilitate identification of new therapeutic targets.

Another parameter that is well hidden within the resection scores, is the impact of residual macroscopic disease on outcomes for both first and repeat CRS/HIPEC operations. In other words, is a single-region $\mathrm{CC} 1$ pelvic resection (remaining disease $<2.5 \mathrm{~mm}$ ) for a high-grade primary, equal to $\mathrm{CC} 1$ resection involving all 13 peritoneal regions, in terms of time to progression, risk of HIPEC failure, and overall survival? We know from perfusion of $1 \mathrm{~mm}$ in size appendiceal cancer organoids, that approximately $20 \%$ of cells will stay alive after $2 \mathrm{~h}$ of heated chemoperfusion. ${ }^{11}$ When the entire extent of the peritoneum is factored in with an average surface of $1.7-2 \mathrm{~m}^{2}$, then the potential of HIPEC failure obtains a more robust dynamic. Is this the time to rethink of what we define as complete cytoreduction, not so much for LGA primaries or epithelioid mesothelioma but for primaries with more aggressive biologic behavior?

The interval between recurrence and initial CRS/HIPEC is used as an indicator of tumor biology. It is well-documented that the longer the interval, the better the survival outcomes, reflecting the selection of patients with favorable tumor biology. ${ }^{12,13}$ How the extent of $\mathrm{CC} 1$ resection impacts the risk of dedifferentiation of a low-grade primary to high grade is unknown. ${ }^{14}$

We have a lot of work to do going forward but for now the take-home message is that complete repeat CRS/ HIPEC is resetting the clock and prolongs survival in LGA and highly selected HGA primaries. Outcomes after repeat CRS predominantly depend on tumor biology and completion of CRS.

DISLCOSURE Konstantinos I. Votanopoulos declares no conflicts of interest.

\section{REFERENCES}

1. Gough DB, Donohue JH, Schutt AJ, Gonchoroff N, Goellner JR, Wilson TO, et al. Pseudomyxoma peritonei. Long-term patient survival with an aggressive regional approach. Ann Surg. 1994;219(2):112-9. doi: https://doi.org/10.1097/00000658-19940 2000-00002.

2. Elias D, Gilly F, Quenet F, Bereder JM, Sideris L, Mansvelt B, et al; Association Francaise de Chirurgie. Pseudomyxoma peritonei: a French multicentric study of 301 patients treated with cytoreductive surgery and intraperitoneal chemotherapy. Eur J Surg Oncol. 2010;36(5):456-62. doi: https://doi.org/10.1016/j.e jso.2010.01.006.

3. Levine EA, Stewart JH 4th, Shen P, Russell GB, Loggie BL, Votanopoulos KI. Intraperitoneal chemotherapy for peritoneal surface malignancy: experience with 1,000 patients. J Am Coll Surg. 2014;218(4):573-85. https://doi.org/10.1016/j.jamcollsurg. 2013.12.013.

4. Votanopoulos KI, Russell G, Randle RW, Shen P, Stewart JH, Levine EA. Peritoneal surface disease (PSD) from appendiceal cancer treated with cytoreductive surgery (CRS) and hyperthermic intraperitoneal chemotherapy (HIPEC): overview of 481 cases. Ann Surg Oncol. 2015;22(4):1274-9. https://doi.org/10.1 245/s10434-014-4147-y.

5. Sugarbaker PH. Cytoreductive surgery and perioperative intraperitoneal chemotherapy: a new standard of care for appendiceal mucinous tumors with peritoneal dissemination. Clin Colon Rectal Surg. 2005;18(3):204-14. https://doi.org/10.1055/s2005-916281.

6. Esquivel J, Sugarbaker PH. Second-look surgery in patients with peritoneal dissemination from appendiceal malignancy: analysis of prognostic factors in 98 patients. Ann Surg. 2001;234(2):198-205.

7. Yan TD, Bijelic L, Sugarbaker PH. Critical analysis of treatment failure after complete cytoreductive surgery and perioperative intraperitoneal chemotherapy for peritoneal dissemination from 
appendiceal mucinous neoplasms. Ann Surg Oncol. 2007;14(8):2289-99. https://doi.org/10.1245/s10434-007-9462-0.

8. Lopez-Ramirez F, Gushchin V, Sittig M, et al. Iterative Cytoreduction and Hyperthermic Intraperitoneal Chemotherapy for Recurrent Mucinous Adenocarcinoma of the Appendix. Ann Surg Oncol. Epub 24 Jan 2022. doi: https://doi.org/10.1245/s10434021-11257-7

9. Votanopoulos KI, Ihemelandu C, Shen P, Stewart JH, Russell GB, Levine EA. Outcomes of repeat cytoreductive surgery with hyperthermic intraperitoneal chemotherapy for the treatment of peritoneal surface malignancy. Journal of the American College of Surgeons. 2012;215(3):412-7. https://doi.org/10.1016/j.jamco 1lsurg.2012.04.023.

10. Choudry HA, Bednar F, Shuai Y, Jones HL, Pai RK, Pingpank JF, Ahrendt SS, Holtzman MP, Zeh HJ, Bartlett DL. Repeat cytoreductive surgery-hyperthermic intraperitoneal chemoperfusion is feasible and offers survival benefit in select patients with peritoneal metastases. Ann Surg Oncol. 2019;26(5):1445-53. https://doi.org/10.1245/s10434-019-07218-w.

11. Forsythe SD, Sasikumar S, Moaven O, Sivakumar H, Shen P, Levine EA, et al. Personalized identification of optimal HIPEC perfusion protocol in patient-derived tumor organoid platform.
Ann Surg Oncol. 2020;27(13):4950-60. https://doi.org/10.1245/ s10434-020-08790-2.

12. Konstantinidis IT, Levine EA, Chouliaras K, Russell G, Shen P, Votanopoulos KI. Interval between cytoreductions as a marker of tumor biology in selecting patients for repeat cytoreductive surgery with hyperthermic intraperitoneal chemotherapy. J Surg Oncol. 2017;116(6):741-5. https://doi.org/10.1002/jso.24703.

13. Mogal H, Chouliaras K, Levine EA, Shen P, Votanopoulos KI. Repeat cytoreductive surgery with hyperthermic intraperitoneal chemotherapy: review of indications and outcomes. J Gastrointest Oncol. 2016;7(1):129-42. https://doi.org/10.3978/j.issn. 2078-6891.2015.131.

14. Mangieri CW, Valenzuela CD, Erali RA, Votanopoulos KI, Shen $\mathrm{P}$, Levine EA. Incidence, risk factors, and outcomes from conversion of low-grade to high-grade appendiceal neoplasms for patients undergoing multiple cytoreductive surgeries with hyperthermic intraperitoneal chemotherapy. Ann Surg Oncol. 2022;29(1):205-11. https://doi.org/10.1245/s10434-021-10660-4.

Publisher's Note Springer Nature remains neutral with regard to jurisdictional claims in published maps and institutional affiliations. 